\title{
2D-3D VASCULAR REGISTRATION BETWEEN DIGITAL SUBTRACTION ANGIOGRAPHIC (DSA) AND MAGNETIC RESONANCE ANGIOGRAPHIC (MRA) IMAGES
}

\author{
H.M. Chan ${ }^{1}$, Albert C.S. Chung ${ }^{1}$, Simon C.H. Yu ${ }^{2}$ and William M. Wells III ${ }^{3}$ \\ ${ }^{I}$ Department of Computer Science, the Hong Kong University of Science and Technology, HK. \\ ${ }^{2}$ Department of Diagnostic Radiology and Organ Imaging, the Prince of Wales Hospital, HK. \\ ${ }^{3}$ Department of Radiology, Brigham and Women's Hospital, Harvard Medical School, Boston, MA, USA.
}

\begin{abstract}
In this paper, we propose a $2 \mathrm{D}-3 \mathrm{D}$ vascular registration algorithm based on an efficient rendering method for generating $2 \mathrm{D}$ projected images from a segmented 3D MRA volume. At the preprocessing stage, vessels are segmented and represented by a number of spheres with centers on the skeleton points of the vessels and radii equal to the distance from the skeleton points to their closest boundary. To generate projected images for 2D-3D registration, instead of using the conventional ray-casting technique, the spheres are projected to the projection plane. The discrepancy between the projected image and the reference DSA image is measured by the sum of squared differences. Experimental results show that our method is computationally efficient. Moreover, based on manually selected markers, it is shown that the accuracies of our proposed method and the conventional ray-casting based registration method are comparable.
\end{abstract}

\section{INTRODUCTION}

Images acquired by different medical imaging modalities provide useful complementary information. For example, magnetic resonance angiographic (MRA) images provide 3D structural information before treatments; digital subtraction angiographic (DSA) images can be acquired in real time and provide 2D structural information during treatments. As such, the registration between 3D MRA and 2D DSA images is very useful for physicians during treatments [1], [2], [3].

Most of the multi-modal registration methods can be broadly classified into two categories: feature-based or intensity-based. In general, the intensity-based method is more accurate. On the other hand, the feature-based method is more computationally efficient [4].

Over the past few years, many intensity-based methods have been introduced, for example, sum of squared differences (SSD), correlation coefficients (CC) and correlation ratio (CR) [5]. The most popular and widely used one is the information-theoretic similarity measure, namely Mutual Information (MI) [6]. It does not require segmentation and only makes use of the assumption of statistical dependence between the intensity values of two images. Recently, Chung and et. al. [7], [8] used a pair of precisely registered or segmented images to build the expected joint (intensity or class) histograms and employed Kullback-Leibler distance to measure the discrepancy between observed and expected joint histograms, and to guide the transformation towards the expected joint histogram.

The feature-based registration algorithms extract features such as surfaces, curves [3] or skeletons [1], [2] at the preprocessing steps. They are faster than intensitybased methods but comparatively less accurate [4]. Also, the extraction of skeletons can be sensitive to noise [9]. Therefore, registration algorithms using skeletons as the only feature for registration will be sensitive to noise as well. Moreover, the surface or curve extraction and representation can be complicated and would also affect the registration accuracy.

\section{OUR 2D-3D REGISTRATION ALGORITHM}

For the conventional ray-casting based 2D-3D registration methods, given the current pose, the projected image of a full intensity 3D MRA volume is generated at each iteration and then compared with the 2D reference DSA image. Generating a maximum intensity projection image with full intensity range by using the ray-casting technique can be very time-consuming [10]. As such, a large portion of the registration computation time would have to be spent on the generations of full intensity MIP images [11]. To shorten the computation time, we propose to use the projected spheres from a segmented MRA volume. 


\subsection{OFFLINE PREPROCESSING OF 3D VOLUME}

Before the registration process, an isotropic volume is built so that the new voxel size is equal to the original inplane voxel size, and the intensities of voxels between slices are estimated by using the trilinear interpolation. Vessels are then segmented by using the global thresholding method and skeletons are extracted with the modified weighted metric $<1, \sqrt{2}, \sqrt{3}>$ [12]. The skeleton points (within a $7 \times 7 \times 7$ window) are connected using the depth first search. The major vessel that we are interested in is usually the connected voxel group containing the largest number of skeleton points. By using the extracted skeleton, the original binary volume can be represented and reconstructed by the set of spheres with the centers set to the coordinates of skeleton points, and radii equal to their distance transform values (which represent distance to the closest boundary) [13].

\subsection{GENERATION OF PROJECTED IMAGE}

In order to mimic the DSA images, the whole projected image is randomly initialized to one of the background intensities of the DSA image. The background intensities of the DSA image can easily be obtained from the boundary pixels.

Given the rigid transformation matrix and the projective geometry, the center of each sphere is projected to the projection plane. The length of its corresponding radius in the projection plane can be calculated based on simple projective geometry. The intensities of all pixels with its distance to the projected center less than the projected radius are assigned to 255 (the max. intensity). Fig. 1 shows a projected image from a binary volume generated by the projection of spheres. Note that a rectangular region of interest containing the major vessels is selected in order to shorten the computation time. Our method is more efficient because it does not need to trace along the projection lines, as required in the ray-casting method. A comparison on computational efficiency between the proposed method and ray-casting method will be presented in Section 3 .

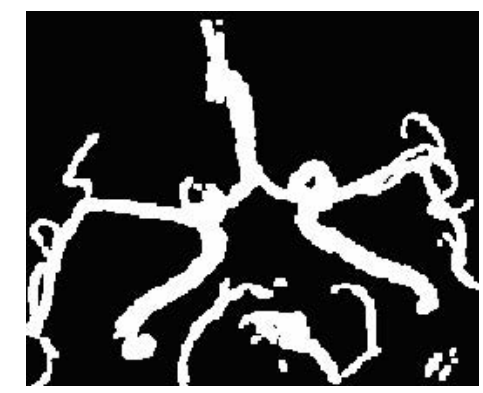

Fig. 1. Projected image from a binary volume generated by the projection of spheres.

\subsection{REGISTRATION PROCESS}

Vessels in both DSA image and projected image have high intensity values (in case the intensities of vessels in DSA image are low, we can simply invert the intensity values of the entire image). The sum of squared differences (SSD) can be employed to measure the difference between the 2D reference image, denoted by $X$, and the binary projected image, denoted by $Y$. The SSD between the two images is given by

$$
\operatorname{SSD}(X, Y)=\sum_{i \in \Phi}(X(i)-Y(i))^{2},
$$

where $i$ and $\Phi$ represent the pixel locations and overlapping image domain respectively. When the two images $X$ and $Y$ are precisely aligned, the value of SSD is expected to be minimum.

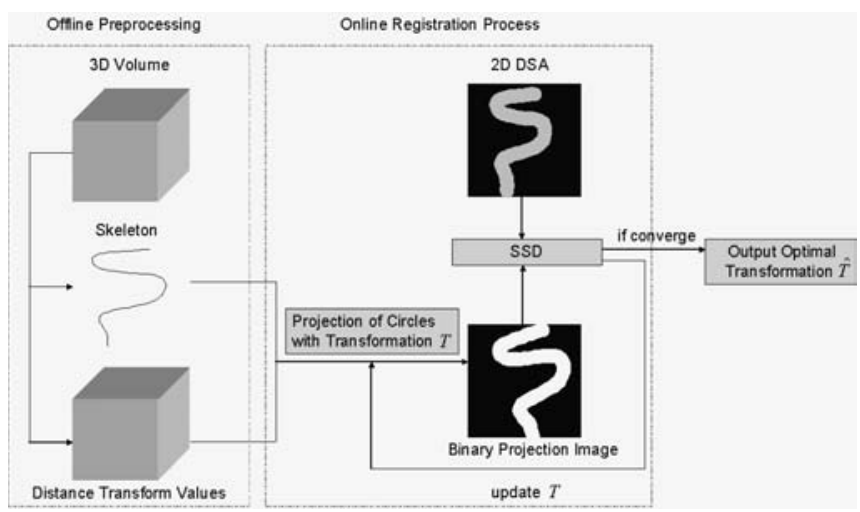

Fig. 2. A flow chart of our registration algorithm.

During the registration process, the floating image is the 3D MRA volume while the reference image is the 2D DSA image, which is fixed in the 3D space. At each iteration, given the current pose, a projected image of the segmented volume is generated by the method mentioned in Section 2.2. The goal is to find the optimal rigid transformation $\hat{T}$ by minimizing the value of SSD (see Equation (1)) between the binary projected image and the 2D reference image,

$$
\hat{T}=\arg \min _{T} \operatorname{SSD}(X, Y(T)) .
$$

Powell's method is used to iteratively search for the minimum SSD value of each parameter (in 3D, the rigid transformation has three translational and three rotational parameters) using Brent's method [14]. The algorithm halts when the percentage change of SSD is below a userspecified threshold. We set this threshold to $0.001 \%$ in our program. The flow chart of our registration algorithm is shown in Fig. 2. 


\section{RESULTS}

In this section, we show the results of the $2 \mathrm{D}-3 \mathrm{D}$ rigid vascular registration algorithm presented in Section 2, and also compare its computational efficiency and accuracy.

We use five pairs of 3D Phase Contrast (PC) MRA and 2D DSA datasets for this experiment. In order to evaluate the performance and accuracy of our registration method, between five and seven target points were chosen by an experienced user using an interactive tool for each case. After the optimal transformation was found, for every user-selected point on a DSA image, the registration error was defined by the Euclidean distance between the selected point and the projected corresponding 3D MRA point. The results of one of the five cases are shown in Fig. 3.

Figs. $3 a$ and $3 b$ show the reference 2D DSA image and the initial alignment of the 3D MRA volume respectively. During the registration, only binary projected images based on spheres were generated and used. The final projected image (at final alignment using the proposed method) is overlaid on the corresponding DSA images (Fig. 3c) and the results are promising. Fig. 3d shows the full intensity maximum intensity projection (MIP) of the registered MRA volumes for visual comparison only.

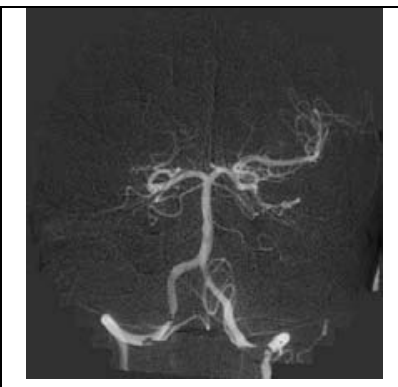

(a) DSA

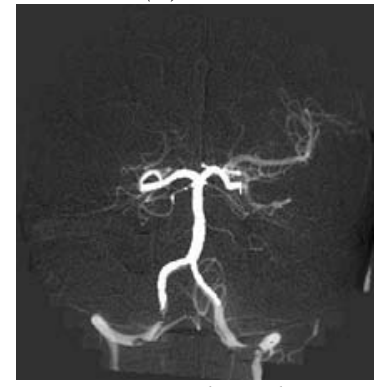

(c) Final result

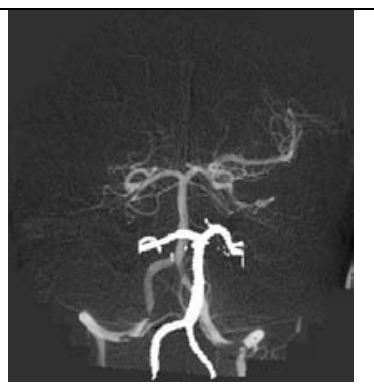

(b) Initial pose

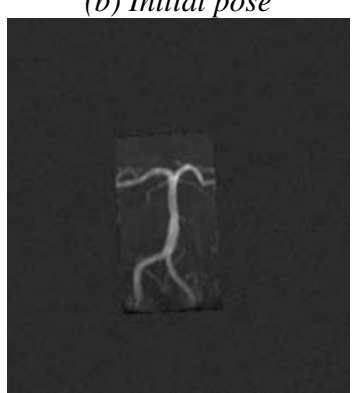

(d) Final result
Fig. 3: Results. (a) DSA image, (b) Initial image alignment and projected spheres, (c) Final image alignment, and binary projected image is overlaid on the DSA, and (d) full intensity MIP of the MRA volume (for visual comparison only).

In order to compare the computation times of generating a projected image from a MRA volume, we implemented the ray-casting method, and generated 1000 full intensity MIP images. The ray-casting method takes 1.34 seconds on average whereas our method, which is based on projected spheres of the segmented MRA volume, takes 0.07 seconds on average, which is about 19 times faster than the ray-casting method.

Table 1 lists the computation times of the whole registration process for (a) the proposed method and (b) the Mutual Information (MI) based 2D-3D registration method based on full intensity ray-casting technique. All experiments done in this paper were run on a $1.7 \mathrm{GHz}$ Pentium IV PC with 768MB of RAM. Based on the results of these five datasets, our method can run faster than the conventional MI-based method by about 18 times.

\begin{tabular}{|c|c|c|c|c|c|c|}
\hline \multirow{2}{*}{ Method } & \multicolumn{7}{|c|}{ Time (sec) } \\
\cline { 2 - 7 } & Case 1 & Case 2 & Case 3 & Case 4 & Case 5 & Mean \\
\hline MI & 233.56 & 425.02 & 460.22 & 768.34 & 352.20 & 447.87 \\
\hline Proposed & 20.21 & 26.07 & 21.76 & 19.81 & 39.86 & 25.54 \\
\hline
\end{tabular}

Table 1: Comparisons of the computation time for the two registration algorithms.

Based on manually selected markers, the mean errors are $1.710 \mathrm{~mm} \pm 0.873$ and $1.992 \mathrm{~mm} \pm 1.061$ for the proposed method and MI-based method respectively. It shows that the registration accuracy of our method is comparable to that of the MI-based method in terms of the mean error values. It also shows that the mean error is less than $2 \mathrm{~mm}$, which is acceptable in this application.

\section{DISCUSSIONS AND CONCLUSIONS}

Preprocessing and segmentation of vessels in DSA images are not required in our method, unlike the methods proposed in the prior work [1], [2], [3], [7] and [8]. During the endovascular treatments, the high acquisition rate (about one image per second) of DSA images makes either manual or semi-automatic segmentation infeasible. Also, automatic segmentation results of DSA images may not always be satisfactory, which will affect the registration accuracy.

In our algorithm, instead of using thinning algorithm [15], skeletons are extracted by using distance transform [12], [13]. With the skeleton points and the distance transform values, the original volume can be recovered exactly [13], which may not be feasible by thinning algorithms. Skeletons extraction is sensitive to noise [6]. Noisy data may give a lot of undesired skeleton points which can affect the accuracy of the method proposed by Kita and et. al. [1], Liu and et. al. [2] and Feldmar and et. al. [3] because they use skeletons alone. Our method requires skeletonization as well, but it does not use skeletons as the only feature. Instead of using skeletons alone, our method also makes use of the distance transform values for generating binary projected images. 
Thus, the accuracy of our registration algorithm is less sensitive to noise.

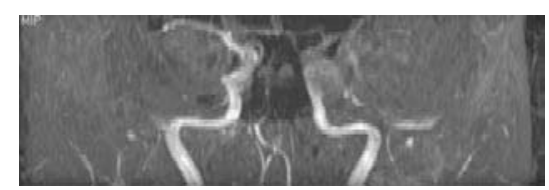

Fig. 4. A maximum intensity projection of TOF-MRA in transverse view.

For Time-Of-Flight (TOF) MRA image volumes, the intensity values of vessels and non-vessels, such as eyeballs, can be similar. The MIP along the transverse view from a TOF-MRA volume is shown in Fig. 4. Some of the vessels are not clearly visible, especially the one at the right hand side. The accuracies of the intensity-based methods may be affected. The non-vessel regions in the MIPs of TOF-MRA may match with the vascular regions in the DSA images. In the proposed method, as segmentation is done and skeleton points are connected, non-vessels will not be projected and the registration accuracy will not be affected by the problem mentioned above.

Instead of projecting all boundary or all vessel voxels, we draw filled circles with centers located at the projected skeleton points, as mentioned in Section 2.2. The reason is that projecting boundary or vessel voxels may result in some blank horizontal or vertical lines in the projected image, which is undesirable and can be avoided by using our method.

To conclude, we have proposed a new multi-modal 2D-3D image registration method based on efficient generation of projected images from a segmented 3D volume. The discrepancy between the projected image and the reference image is measured by the sum of squared differences as similarity measure. Experimental results show that our method is efficient, while the accuracies are comparable with those of the conventional ray-casting based $2 \mathrm{D}-3 \mathrm{D}$ registration method.

\section{ACKNOWLEDGMENTS}

We would like to thank A. Norbash at the Department of Neuroradiology, Brigham and Women's Hospital, Harvard Medical School, USA for providing data. This work was supported in part by DAG01/02.EG04, SSRI01/02.EG22 and HKUST6209/02E.

\section{REFERENCES}

[1] Y. Kita, D.L. Wilson and Noble J. Alison, "Realtime Registration of 3D Cerebral Vessels to X-ray Angiograms", MICCAI'97, pp.1125-1133.

[2] A. Liu, E. Bullitt and S.M. Pizer, "3D/2D registration via skeletal near projective invariance in tubular objectives", MICCAI'98, pp.952-963.
[3] J. Feldmar, G. Malandain, N. Ayache, S. FernándezVidal, E. Maurincomme and Y. Trousset, "Matching 3D MR Angiography Data and 2D X-ray Angiograms", CVRMed-MRCAS'97, Grenoble, France, pp.129-138,.

[4] R.A. McLaughlin, J. Hipwell, D.J. Hawkes, J.A. Noble, J.V. Byrne and T. Cox, "A comparison of 2D-3D intensity-based registration and feature-based registration for neurointerventions", MICCAI'02, pp.517-24.

[5] J.P.W. Pluim, J.B.A. Maintz and M.A. Viergever, "Mutual-information-based registration of medical images: a survey", TMI, 22(8):986-1004, 2003.

[6] W. Wells, P. Viola, H. Atsumi, S. Nakajima and R. Kikinis, "Multi-modal Volume Registration by Maximization of Mutual Information", Medical Image Analysis, 1(1):32-52, 1996.

[7] A.C.S. Chung, W.M. Wells III, A. Norbash, W.E.L. Grimson, "Multi-modal Image Registration by Minimising Kullback-Leibler Distance", MICCAI'02, pp. 525-532.

[8] H.M. Chan, A.C.S. Chung, S.C.H. Yu, A. Norbash, W.M. Wells III, "Multi-modal image registration by minimizing Kullback-Leibler distance between expected and observed joint class histograms", CVPR'03, Vol. 2, pp. 570-576.

[9] R. Fisher, S. Perkins, A. Walker and E. Wolfart, http://www.dai.ed.ac.uk/HIPR2/skeleton.htm, 2000.

[10] A. Watt, M. Watt, Advanced Animation and Rendering Techniques: Theory and Practice, Addison-Wesley, Reading, Massachusetts, 1992.

[11] E. Cosman, "Rigid Registration of MR and Biplanar Fluoroscopy", Masters Thesis, Dept. of Electrical Engineering and Computer Engineering, MIT, 2000.

[12] N. Gagvani, "Skeletons and Volume Thinning in Visualization", MS. Thesis, Rutgers University, New Brunswick, New Jersey, 1997.

[13] A. K. Jain, Fundamentals of Digital Image Processing, p.381-389, Prentice Hall, 1989.

[14] W.H. Press, B.P. Flannery, S.A. Teukolsky, W.T. Vetterling, Numerical Recipes in $C$, 2nd Edition, Cambridge University Press, p.402-405,412-420; Kluwer Academic Publisher, 1995.

[15] K. Palgyi, E. Sorantin, E. Balogh, A. Kuba, C. Halmai, B. Erdohelyi, and K. Hausegger, "A Sequential 3D Thinning Algorithm and Its Medical Applications", IPMI'01, pp. 409-415. 